\title{
Quantum Manipulation of Trapped Ions in Two Dimensional Coulomb Crystals
}

\author{
D. Porras and J. I. Cirac \\ Max-Planck-Institut für Quantenoptik, Hans-Kopfermann-Straße 1, Garching, D-85748, Germany
}

(Received 23 January 2006; published 29 June 2006)

\begin{abstract}
We show that a large number of ions forming a 2D Coulomb crystal provides an almost ideal system for scalable quantum computation and quantum simulation. In particular, the coupling of the internal states to the motion of the ions transverse to the crystal plane allows one to implement two-qubit quantum gates. We analyze in detail the decoherence induced by anharmonic couplings, and show that very high gate fidelities can be achieved with current experimental setups.
\end{abstract}

DOI: 10.1103/PhysRevLett.96.250501

PACS numbers: 03.67.Lx, 03.67.Pp, 42.50.Vk

The search for a physical system where quantum computation is feasible is at the focus of an intense theoretical and experimental activity [1]. Ion traps are by now among the most promising candidates for a many-qubit quantum processor. In this system, qubits are stored in internal electronic states, and collective vibrational modes of the ions allow us to induce quantum gates between them [2]. Following this idea, the building blocks for quantum computation have already been demonstrated in experiments with a few qubits [3]. Most of the current efforts to scale up the size of current ion quantum processors rely on the fabrication of arrays of microtraps [4], in which a large number of ions can be stored and shuttled. Even though an astonishing progress has been achieved in this direction in the last years, the scalability of this system still demands technical advances in microfabrication and trap design [5].

Penning traps provide us with an alternative trapping scheme, where a large number of ions $\left(10^{4}-10^{6}\right)$ can be confined by a potential with approximate cylindrical symmetry [6]. Axial confinement is induced by a static electric field, whereas radial confinement is a result of the rotation of the ions under an axial magnetic field. If the axial confinement is strong enough, ions arrange themselves in a triangular lattice on a single plane, which corresponds to a classical two-dimensional (2D) Wigner crystal. The appeal of this system lies on the fact that ions are naturally ordered in a 2D regular array, without the need of individual micropotentials. Furthermore, ions are separated by distances of the order of tens of microns, such that they are individually addressable by optical means [7]. Thus, ions in Penning traps may appear as ideally suited for quantum computation and quantum simulation. However, this system has never been considered for this task [8]. First, because the complicated vibrational level structure of the crystal makes it difficult to apply here schemes that require resolution of single vibrational modes. In addition to that, typical schemes usually rely on the coupling of qubits to modes in directions parallel to the crystal. In current experiments with Penning traps, Doppler cooling of ions has reached temperatures of at most $1 \mathrm{mK}$, which implies occupation numbers of $10^{2}-10^{3}$ in the in-plane vibrational modes, so that it seems not to be possible to use them for quantum operations.

In this Letter we show how to circumvent these problems by exploiting the ions' motion along the axial direction (Fig. 1). This approach benefits from the high axial confinement frequencies (and thus smaller occupation numbers at finite temperature), as well as from the fact that ions are weakly coupled in this direction, something that enormously simplifies the description of the ions' motion. In particular, we show how it is possible to carry out two-qubit gates between ions with high fidelities by performing a careful analysis of the main sources of decoherence. We emphasize that the results derived here also imply that this system is ideally suited for quantum simulations, which may be especially interesting due to the fact that ions are displayed in a triangular structure and favor the simulation of magnetic frustrated systems.

The main source of decoherence in our scheme is due to the anharmonic terms in the Coulomb interaction, which induce a coupling between axial motion and in-plane hot vibrational modes, and lead to a residual qubit-phonon coupling. The description of such decoherence poses an involved theoretical problem, because of the large number of vibrational modes that participate in the process. However, it gets simplified due to the fact that the environ-

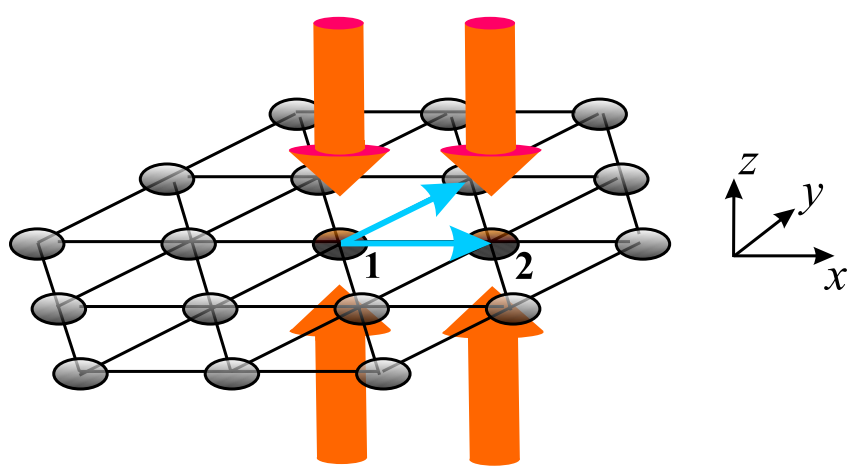

FIG. 1 (color online). Quantum gate in a 2D Coulomb crystal: standing waves induce a state-dependent dipole force on two nearest neighbors in a triangular lattice. 
ment is in a Gaussian state, which allows us to simplify the calculation of correlation functions that appear in finitetemperature, time-dependent perturbation theory. We show that in a range of parameters where axial confinement is large enough, the error induced in the quantum gate is very small. Furthermore, the adjustment of the gate time allows us to correct for the influence of the phonon environment, and decrease the gate error by more than 1 order of magnitude.

Let us consider a system of $N$ ions forming a 2D Coulomb crystal. We study, for concreteness, the performance of a "pushing gate" [9] in the axial direction, an approach that has the advantage that single vibrational modes do not have to be resolved, and the gate can operate at finite temperature. Since we are interested in estimating the consequences of decoherence, we neglect finite size effects, and describe the crystal by a triangular lattice with periodic boundary conditions. Let us assign the $\mathbf{z}$ direction to the axis of the trap, such that ions occupy equilibrium positions in the $\mathbf{x}-\mathbf{y}$ plane:

$$
\mathbf{R}_{\mathbf{r}}^{0}=\left(r_{1} \mathbf{a}_{1}+r_{2} \mathbf{a}_{2}\right) d_{0}, \quad r_{j}=1, \ldots, L,
$$

where $\mathbf{a}_{1}=(1,0,0), \mathbf{a}_{2}=(1 / 2, \sqrt{3} / 2,0)$, and $d_{0}$ is the distance between ions. The potential is given by a trapping term, plus the Coulomb repulsion:

$$
\begin{aligned}
V & =V_{\text {Trap }}+V_{\text {Coul }}, \\
V_{\text {Coul }} & =\frac{1}{2} \sum_{\mathbf{r}, \mathbf{s}} \frac{e^{2}}{\left|\mathbf{R}_{\mathbf{r}}^{0}-\mathbf{R}_{\mathbf{s}}^{0}+\mathbf{R}_{\mathbf{r}}-\mathbf{R}_{\mathbf{s}}\right|} .
\end{aligned}
$$

$\mathbf{R}_{\mathbf{r}}=\left(X_{\mathbf{r}}, Y_{\mathbf{r}}, Z_{\mathbf{r}}\right)$, are the ions' coordinates with respect to the equilibrium positions, and $V_{\text {Trap }}$ is the harmonic trapping potential, with frequencies $\omega_{j}$ in each spatial direction, $j=x, y, z$. Note that in a Penning trap, (2) corresponds to the potential in a frame rotating with the ion crystal [10].

In the harmonic approximation, $V_{\text {Coul }}$ is expanded up to second order in $\mathbf{R}_{\mathbf{r}}$, and the axial (z) and in-plane $(\mathbf{x}, \mathbf{y})$ modes are independent. The vibrational harmonic Hamiltonian is given by $H_{\mathrm{vib}}^{(0)}=\sum_{\lambda, \mathbf{q}} \hbar \omega_{\mathbf{q}}^{\lambda} a_{\mathbf{q}, \lambda}^{\dagger} a_{\mathbf{q}, \lambda} \cdot \mathbf{q}$ is the phonon wave vector, and $\lambda$ runs over the three possible polarizations: $\lambda=z$ (axial modes), and the two in-plane modes $(\lambda=\|, \perp)$ corresponding to longitudinal and transverse modes. Local displacements can be expressed in terms of collective coordinates:

$$
\begin{aligned}
& \mathbf{R}_{\mathbf{r}}=(1 / \sqrt{N}) \sum_{\lambda, \mathbf{q}} \mathbf{e}_{\mathbf{q}}^{\lambda} R_{\mathbf{q}}^{\lambda} e^{i \mathbf{q r},}, \\
& R_{\mathbf{q}}^{\lambda}=\sqrt{\hbar / 2 m \omega_{\mathbf{q}}^{\lambda}}\left(a_{\mathbf{q}, \lambda}^{\dagger}+a_{\mathbf{q}, \lambda}\right),
\end{aligned}
$$

where $\mathbf{e}_{\mathbf{q}}^{\lambda}$ are the polarization vectors, which are eigenstates of the Fourier transform of the harmonic ion-ion interaction [11]:

$$
\begin{aligned}
\Omega_{\mathbf{q}}^{i, j} & =\delta_{i, j} \omega_{j}^{2}+\sum_{\mathbf{s}} \frac{e^{2}}{m}[1-\cos (\mathbf{s q})] V_{\mathbf{s}}^{i, j}, \\
V_{\mathbf{s}}^{i, j} & =\frac{1}{\left|\mathbf{R}_{\mathbf{s}}^{0}\right|^{3}}\left(\frac{3\left(\mathbf{R}_{\mathbf{s}}^{0}\right)_{i}\left(\mathbf{R}_{\mathbf{s}}^{0}\right)_{j}}{\left|\mathbf{R}_{\mathbf{s}}^{0}\right|^{2}}-\delta_{i, j}\right) .
\end{aligned}
$$

For each $\mathbf{q}, \omega_{\mathbf{q}}^{\lambda}$ are given by the eigenvectors of the $3 \times 3$ matrix $\sqrt{\Omega_{\mathbf{q}}}$.

The "pushing gate" works by coupling the internal states of two ions, which we label 1 and 2 , to the axial (z) motion [9,12]. An off-resonant standing-wave induces a force which displaces the position of ions 1 and 2, in a direction which depends on their internal state [13] (Fig. 1):

$$
H_{\mathrm{f}}(t)=\sum_{j=1,2} F(t) Z_{\mathbf{r}_{j}} \sigma_{j}^{z}
$$

where $\sigma_{j}^{z}$ are operators acting on the internal states of ions at sites $\mathbf{r}_{j}$. To simplify our calculations, we consider the following time-dependent force: $F(t)=F e^{-i \Gamma|t| / 2}$ $(-\infty<t<\infty)$. However, our results are qualitatively valid for other pulse shapes with gate time $1 / \Gamma$. Trapping parameters are chosen such that $\beta_{z}=e^{2} / m \omega_{z}^{2} d_{0}^{3} \ll 1$ [13]. In this limit, ions moving in the axial direction can be considered as independent harmonic oscillators weakly coupled by the Coulomb interaction, akin to the case of microtrap arrays. Furthermore, we will be in the adiabatic limit, defined here by:

$$
\mathcal{E}_{z}=8\left(\Gamma / \omega_{z}\right)^{2}\left(F Z_{0} / \hbar \omega_{z}\right)^{2}\left(2 \bar{n}_{z}+1\right) \ll 1,
$$

where $Z_{0}$ is the axial ground state size, and $\bar{n}_{z}$ is the mean axial phonon number. $\mathcal{E}_{z}$ is, indeed, the error induced in the quantum gate by nonadiabatic effects in the axial modes [12]. Under condition (6), internal states end up being decoupled from the axial motion after the gate, and follow the unitary evolution given by:

$$
U_{\mathrm{g}}=e^{-i \int d t J(t) \sigma_{1}^{z} \sigma_{2}^{z}}, \quad J(t)=2 \beta_{z}\left[F(t) Z_{0} / \hbar \omega_{z}\right]^{2} \omega_{z}
$$

In the following we consider the choice $\Gamma=J(0) \pi / 8$, such that $U_{\mathrm{g}}$ corresponds, up to local operations, to a sign gate.

Unfortunately, the axial motion is coupled to the inplane modes by anharmonic terms in $V_{\text {Coul }}$, being the lowest order ones of the form $X Z^{2}, X^{2} Z^{2}$, that is, quadratic in the axial coordinates. Since resonances between axial and in-plane vibrational frequencies lead to divergences in the correlation functions of these terms, the effect of anharmonicities is reduced if $\omega_{\mathbf{q}}^{z} \gg 2 \omega_{\mathbf{q}}^{\|, \perp}$. The axial vibrational bandwidth is proportional to $\beta_{z}$, and, thus, this condition can be imposed by choosing a tight enough axial confinement [see [14]]. In the case of $N=10^{4}$ ions, $\omega_{z} \approx$ $50 \omega_{\mathrm{xy}}$ is enough to ensure this condition [see Fig. 2(a)], so 

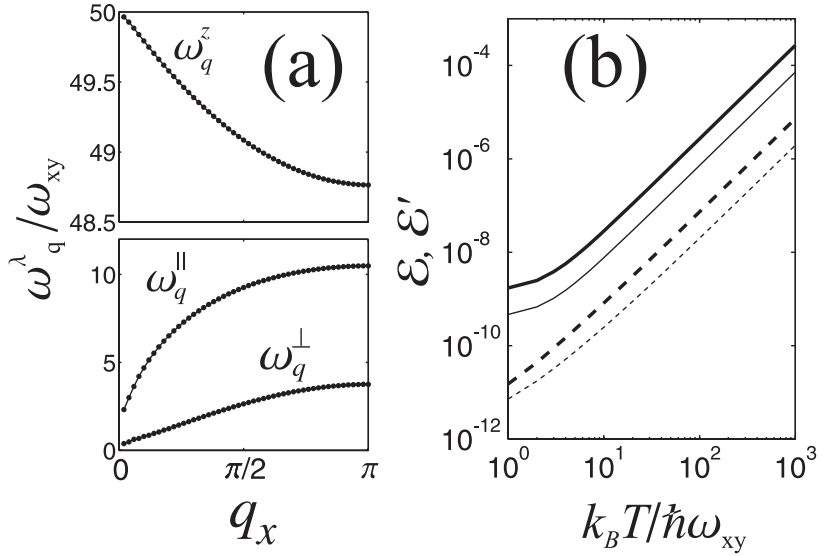

FIG. 2. (a) Spectrum of the vibrational modes with wave vector $\mathbf{q}$ along the $\mathbf{x}$ direction. $N=10^{4}$ ions, $\omega_{z}=50 \omega_{\mathrm{xy}}$, and we consider periodic boundary conditions. (b) Continuous lines: $\mathcal{E}$ (error without phase correction). Dashed lines: $\mathcal{E}^{\prime}$ (error with correction of the gate time). Thick lines and thin lines correspond to $\omega_{\mathrm{xy}}=20 \mathrm{kHz}$ and $\omega_{\mathrm{xy}}=200 \mathrm{kHz}$, respectively. $N=10^{4}$ ions and $\Gamma / \omega_{\mathrm{xy}}=0.05$.

that we will assume this ratio in all the examples presented along this work.

Let us now quantify the loss of fidelity induced by anharmonic couplings. The force (5) displaces the ions in the axial direction by $\bar{Z}(t)=2 F(t) Z_{0}^{2} / \hbar \omega_{z}$. In the limit (6), ions 1 and 2 follow adiabatically the displacement induced by the force, so that one can neglect the fluctuations in the coordinates $Z_{\mathbf{r}_{j}}$, and replace them by their ground state average, $\bar{Z}(t) \sigma_{j}^{z}$, when computing anharmonic corrections. The anharmonic energy dependence on the ions' position results in the following coupling between internal states and in-plane modes:

$$
\begin{aligned}
H_{\mathrm{xy}}^{\mathrm{dec}}(t) & =H_{\mathrm{xy}}^{\mathrm{ah}}(t) \frac{1}{4}\left(\sigma_{1}^{z}-\sigma_{2}^{z}\right)^{2}, \\
H_{\mathrm{xy}}^{\mathrm{ah}}(t) & =\bar{Z}(t)^{2}\left(\mathbf{A}^{\mathrm{T}} \mathbf{R}_{1,2}+\mathbf{R}_{1,2}^{\mathrm{T}} \mathbf{B R}_{1,2}\right) .
\end{aligned}
$$

$\mathbf{R}_{1,2}\left(\mathbf{R}_{1,2}^{0}\right)$ is the vector given by the in-plane components of $\mathbf{R}_{\mathbf{r}_{1}}-\mathbf{R}_{\mathbf{r}_{2}}\left(\mathbf{R}_{\mathbf{r}_{1}}^{0}-\mathbf{R}_{\mathbf{r}_{2}}^{0}\right)$. A and $\mathbf{B}$ are third and fourth order anharmonic Coulomb interaction terms, respectively:

$$
\mathbf{A}=3 \frac{e^{2}}{d_{0}^{5}} \mathbf{R}_{1,2}^{0}, \quad \mathbf{B}=\frac{3 e^{2}}{d_{0}^{5}}\left(\mathbf{1}-\frac{5}{2} \frac{\mathbf{R}_{1,2}^{0}\left(\mathbf{R}_{1,2}^{0}\right) T}{d_{0}^{2}}\right) .
$$

Note that $H_{\mathrm{xy}}^{\mathrm{dec}}$ excites the in-plane phonons depending on the ions' internal states, and, thus, entangles the qubits with the environment.

Assume that the internal states are initially in a pure state: $|\Psi\rangle=\sum_{\alpha} c_{\alpha}|\alpha\rangle(|\alpha\rangle=|00\rangle,|01\rangle,|10\rangle,|11\rangle)$, such that the initial density matrix of the total system is given by: $\rho_{i}=|\Psi\rangle\langle\Psi| \otimes \rho_{\mathrm{xy}}^{0}$, with $\rho_{\mathrm{xy}}^{0}$, a thermal phonon state. After the action of the quantum gate, the final density matrix is

$$
\rho_{f}=\sum_{\alpha, \beta} c_{\alpha} c_{\beta}^{*}\left(U_{g}|\alpha\rangle\langle\beta| U_{g}^{\dagger}\right)\left[U_{\mathrm{xy}}^{\alpha} \rho_{\mathrm{xy}}^{0}\left(U_{\mathrm{xy}}^{\beta}\right)^{\dagger}\right],
$$

where $U_{\mathrm{xy}}^{\alpha}$, is the evolution operator of the in-plane phonons, $U_{\mathrm{xy}}$, projected in the internal state $\alpha$. In (10) we have assumed that the axial modes can be described classically in $H_{\mathrm{xy}}^{\mathrm{dec}}$ due to the adiabaticity of the quantum gate, such that we can factorize the evolution operator. We define the (worst-case) reduced fidelity, $\mathcal{F}$, of the quantum gate by the overlap of the qubits' reduced density matrix obtained from (10), with the qubit quantum state after a perfect gate, minimized over all possible two-qubit initial states. Note that $U_{\mathrm{xy}}^{00}=U_{\mathrm{xy}}^{11}=U_{\mathrm{xy}}^{[0]}$, and $U_{\mathrm{xy}}^{01}=U_{\mathrm{xy}}^{10}=U_{\mathrm{xy}}^{[\mathrm{ah}]}$, where $U_{\mathrm{xy}}^{[0]}$ and $U_{\mathrm{xy}}^{[\mathrm{ah}]}$, are the in-plane phonon evolution operator in the absence and presence of anharmonic couplings, respectively. Thus, the fidelity is completely determined by the following complex quantity $\overline{\mathcal{F}}$ :

$$
\begin{aligned}
\overline{\mathcal{F}} & =\operatorname{tr}\left\{|01\rangle\langle 00| U_{\mathrm{xy}} \rho_{i}\left(U_{\mathrm{xy}}\right)^{\dagger}\right\}=\operatorname{tr}_{\mathrm{xy}}\left\{U_{\mathrm{xy}}^{[\mathrm{ah}]} \rho_{\mathrm{xy}}^{0} U_{\mathrm{xy}}^{[0] \dagger}\right\} \\
& =\operatorname{tr}_{\mathrm{xy}}\left\{\mathcal{T} \exp \left[-i \int_{-\infty}^{\infty} H_{\mathrm{xy}}^{\mathrm{ah}}(\tau) d \tau\right] \rho_{\mathrm{xy}}^{0}\right\},
\end{aligned}
$$

which corresponds to the mean value of the evolution operator $U_{\mathrm{xy}}^{[\mathrm{ah}]}$, in the interaction picture with respect to $H_{\text {xy }}^{\text {ah }}$ [16]. The worst-case error is $\mathcal{E}=1-\mathcal{F}=$ $[1-\mathfrak{R}(\overline{\mathcal{F}})] / 2$. However, note that the fidelity can be improved, since, according to Eq. (8), the spin-phonon coupling depends on the operator $\left(1-\sigma_{1}^{z} \sigma_{2}^{z}\right)$. For this reason, a correction of the gate time allows us to cancel the phase of $\overline{\mathcal{F}}$, and define $\mathcal{E}^{\prime}=(1-|\overline{\mathcal{F}}|) / 2$, which quantifies the worst-case error after the correct calibration of the gate duration.

Equation (11) is a good starting point for perturbation theory, which can be carried out by expanding the timeordered exponential. First of all, let us study the scaling of the terms appearing in $H_{\mathrm{xy}}^{\mathrm{ah}}$, and check whether a peturbative approach is indeed justified. The anharmonic coupling (8) can be rewritten in terms of collective variables in the interaction picture:

$$
H_{\mathrm{xy}}^{\mathrm{ah}}(\tau)=\sum_{\lambda, \mathbf{q}} F_{\mathbf{q}}^{\lambda}(\tau) R_{\mathbf{q}}^{\lambda}(\tau)+\sum_{\lambda, \lambda^{\prime}, \mathbf{q}, \mathbf{k}} G_{\mathbf{q} \mathbf{k}}^{\lambda \lambda^{\prime}}(\tau) R_{\mathbf{q}}^{\lambda}(\tau) R_{\mathbf{k}}^{\lambda^{\prime}}(\tau) .
$$

According to Eqs. (7)-(9), each term scales like:

$$
\begin{aligned}
\overline{F_{\mathbf{q}}^{\lambda} R_{\mathbf{q}}^{\lambda}} & \approx J \frac{X_{0}}{d_{0}} \approx \Gamma\left(\frac{X_{0}}{d_{0}}\right), \\
\overline{G_{\mathbf{q}, \mathbf{k}}^{\lambda, \lambda^{\prime}} R_{\mathbf{q}}^{\lambda} R_{\mathbf{k}}^{\lambda^{\prime}}} & \approx J\left(\frac{X_{0}}{d_{0}}\right)^{2} \approx \Gamma\left(\frac{X_{0}}{d_{0}}\right)^{2},
\end{aligned}
$$

where $X_{0}$ is the size of the ground state in the radial trapping potential, and by $\bar{O}$, we mean the square root of the variance of the operator in the ground state. Since the evolution of the in-plane modes is governed by the in-plane trapping frequency, $\omega_{\mathrm{xy}}$, we expect that terms in 
perturbation theory scale according to $\Gamma / \omega_{x y}$ in Eq. (13). We study two sets of experimental parameters with $\omega_{\mathrm{xy}}=20(200) \mathrm{kHz}$, and $\omega_{z}=1(10) \mathrm{MHz}$, which implies $d_{0}=46.8(10.1) \mu \mathrm{m}$. Let us consider $N=10^{4}$ ions, such that both values lead to $\beta_{z}=3.8 \times 10^{-3}$. Typical temperatures of $T=1 \mathrm{mK}$ can be reached after Doppler cooling, such that mean phonon numbers are $\bar{n}_{z} \approx 20(2)$ in the axial modes, and $\bar{n}_{\mathrm{xy}} \approx 10^{3}\left(10^{2}\right)$, in the center of mass in-plane modes. If we choose $\left(F Z_{0} / \hbar \omega_{z}\right)=0.234$, such that $\mathcal{E}_{z}<$ $10^{-5}$, we get $\Gamma / \omega_{\text {xy }}=5 \times 10^{-2}$. Taking into account that $X_{0} / d_{0}$ is small (as it should be, if the assumption of harmonic vibrational modes is valid), all terms are small in (13). In particular, $X_{0} / d_{0}=3.6 \times 10^{-3}$, and $5.0 \times$ $10^{-3}$, in the cases $\omega_{\mathrm{xy}}=20$, and $200 \mathrm{kHz}$, respectively.

To quantify precisely the error in the quantum gate, we proceed as follows. See the appendix of Ref. [17] for details. We expand the time-ordered exponential (11) up to fourth order in $H_{\mathrm{xy}}^{\mathrm{ah}}$, and keep all the terms up to order $\left(X_{0} / d_{0}\right)^{4}$. Each contribution can be expressed in terms of time integrations of correlation functions of collective coordinates $R_{\mathbf{q}}^{\lambda}$. The problem gets numerically tractable by application of the Wick's theorem, which allows us to express real time high order correlation functions in terms of the two-operator correlation function. Note that Wick's theorem can be applied here, even when we are at finite temperatures, because of the statistical properties of the phonon thermal (Gaussian) state. Finally, a summation of sets of diagrams up to infinite order is done by means of the linked cluster theorem, such that (11) takes finally the form of the exponential of low order irreducible diagrams [18].

The results of our calculation are presented in Fig. 2(b), where we show our results for the two sets of experimental parameters discussed above. Note that at high temperatures, like those ones that occur in current experiments with Penning traps, we get a quadratic dependence with temperature, which can be explained by the dominant contribution of terms that are second order in the coupling $G_{\mathbf{q}, \mathbf{k}}^{\lambda, \lambda^{\prime}}$. These terms have the form $\left\langle R_{\mathbf{q}}^{\lambda}\left(t_{1}\right) R_{\mathbf{k}}^{\lambda^{\prime}}\left(t_{1}\right) R_{-\mathbf{q}}^{\lambda}\left(t_{2}\right) R_{-\mathbf{k}}^{\lambda^{\prime}}\left(t_{2}\right)\right\rangle$, and diverge in the limit of large times when the resonance condition $\omega_{\mathbf{q}}^{\lambda}=\omega_{\mathbf{k}}^{\lambda^{\prime}}$ is met. The correction of the phase by adjustment of the gate time allows us to reduce the error by more than 1 order of magnitude. Even with the highest temperatures considered here, which correspond to the limit of Doppler cooling, anharmonic terms induce very small errors. With the range of parameters considered in this work, we get rates $\Gamma=1$, $10 \mathrm{kHz}$. A limitation in the number of quantum gates due to heating reduces the number of gates to a maximum of $\approx 10^{2}$, with current heating rates. However, this quantity is amenable to be improved by increasing the quality of the vacuum [19], or by sympathetic cooling with spectator ions [4].

Finally, our proposal could also be used for the quantum simulation of interacting spin systems, by applying a state- dependent force to all the ions at the same time. In this way, as shown in Ref. [20], an antiferromagnetic Ising interaction is induced between the internal states, which behave like effective spins. If we add a transverse field of the form $(\Omega / 2) \sum_{\mathbf{r}} \sigma_{\mathbf{r}}^{x}$, by means of a global carrier transition, then this experimental setup allows us to study the rich phenomenology of quantum frustration in triangular lattices [21]. Note that our analysis of the decoherence induced by low-energy vibrational modes also implies the viability of this approach, since the effective spin-spin interactions are an always-on version of the qubit-qubit coupling induced during the quantum gate.

We thank J. Bollinger, D. Leibfried, and M. Aguado for interesting discussions. Work supported by CONQUEST, SCALA, and Marie Curie under Contract No. MEIF-2004010350.

[1] J. I. Cirac and P. Zoller, Phys. Today 57, No. 3, 38 (2004).

[2] J. I. Cirac and P. Zoller, Phys. Rev. Lett. 74, 4091 (1995).

[3] C. Monroe et al., Phys. Rev. Lett. 75, 4714 (1995); D. Leibfried et al., Nature (London) 422, 412 (2003); F. Schmidt-Kaler et al., Nature (London) 422, 408 (2003).

[4] D. Kielpinksi, C. Monroe, and D. J. Wineland, Nature (London) 417, 709 (2002); D. Stick et al., Nature Phys. 2, 36 (2006).

[5] T. S. Metodi et al., quant-ph/0509051.

[6] W. M. Itano et al., Science 279, 686 (1998); T. B. Mitchell et al., Science 282, 1290 (1998).

[7] T. B. Mitchell et al., Phys. Rev. Lett. 87, 183001 (2001).

[8] Note that arrays of single electron Penning traps have been proposed for quantum arrays; see G. Ciaramicoli et al., Phys. Rev. Lett. 91, 017901 (2003).

[9] J. I. Cirac and P. Zoller, Nature (London) 404, 579 (2000).

[10] We study here the limit in which the cyclotron and rotation frequencies satisfy the relation $\Omega_{c} / 2=\omega_{\mathrm{md}}$, in which there is no magnetic field in the rotating frame.

[11] D. Dubin, Phys. Rev. Lett. 71, 2753 (1993).

[12] T. Calarco, J.I. Cirac, and P. Zoller, Phys. Rev. A 63, 062304 (2001); M. Sasura and A. M. Steane, Phys. Rev. A 67, 062318 (2003).

[13] The coupling in Eq. (5) can be obtained with a configuration of lasers and polarizations similar to that one presented by D. J. Wineland et al., quant-ph/0212079.

[14] $\beta_{z}$ scales like $\left(\omega_{\mathrm{xy}} / \omega_{z}\right)^{2} \sqrt{N}$ [15]; thus, it can be tuned by choosing the axial or radial trapping frequency ratio.

[15] D. Dubin and T. O’Neil, Rev. Mod. Phys. 71, 87 (1999).

[16] M. Znidaric and T. Prozen, J. Phys. A 36, 2463 (2003).

[17] D. Porras and J. I. Cirac, quant-ph/0601148.

[18] J.W. Negele and H. Orland, Quantum Many-Particle Systems (Addison-Wesley, New York, 1988).

[19] M. J. Jensen, T. Hasegawa, and J. Bollinger, Phys. Rev. A 70, 033401 (2004).

[20] D. Porras and J. I. Cirac, Phys. Rev. Lett. 92, 207901 (2004); X.-L. Deng, D. Porras, and J. I. Cirac, Phys. Rev. A 72, 063407 (2005).

[21] G. H. Wannier, Phys. Rev. 79, 357 (1950); R. Moessner and S. L. Sondhi, Phys. Rev. B 63, 224401 (2001). 